\title{
MÉTODO MULTICRITERIO PARA LA TOMA DE DECISIONES CON UN ENFOQUE DIFUSO: UNA APLICACIÓN EN LA AVIACIÓN
}

\section{FUZZY MULTICRITERIA DECISION MAKING APPROACH: AN APPLICATION IN AVIATION INDUSTRY}

\author{
Ing. María Cecilia Vásquez Carrillo, PhD. Rocco Tarantino Alvarado. \\ Universidad de Pamplona. Facultad de Ingenierías y Arquitectura. \\ Ciudadela Universitaria. Pamplona, Norte de Santander, Colombia. \\ Tel.: (+577) 568 5303, Fax: (+577) 568 5303, Ext. 144. \\ E-mail: winchechi_8@ hotmail.com,rocco.tarantino@gmail.com
}

\begin{abstract}
Resumen: Para un aeropuerto en fase de desarrollo, la planeación estratégica juega un papel importante y la administración aprovecha para asignar los recursos disponibles a aquellas áreas con mayor prioridad o relevancia dentro del mismo. Este artículo presenta una aplicación del método análisis jerárquico de procesos difuso, con el fin de cuantificar la importancia de ciertos componentes que generan mayor impacto dentro de la terminal de pasajeros del Aeropuerto Internacional Camilo Daza, y de esta manera plantear estrategias para el mejoramiento continuo.
\end{abstract}

Palabras clave: Toma de decisiones multicriterio, conjuntos difusos, planeación, aeropuertos.

\begin{abstract}
For an airport in the development phase, strategic planning plays an important role and management uses to allocate available resources to those areas with the highest priority or relevance within it. This paper presents an application of hierarchical analysis method of fuzzy processes, in order to quantify the importance of certain components that generate greater impact within the passenger terminal Camilo Daza International Airport, and thus propose strategies for continuous improvement.
\end{abstract}

Keywords: Multicriteria decision making, fuzzy sets, planning, airports.

\section{INTRODUCCIÓN}

El impacto económico que generan los aeropuertos en cada zona, ha hecho que se posicionen en el primer lugar de importancia en las actividades turísticas y de negocios de cada región del país.

Anteriormente, los principales gobiernos del mundo han aunado esfuerzos para la innovación y el fortalecimiento de cada terminal, con base en los estándares internacionales, en miras al mejoramiento de la comunicación y el transporte, tanto de mercancías, como de personas alrededor del mundo.
Uno de sus componentes esenciales está conformado por las políticas de atención al cliente, lo cual ha sido foco de atención de varias organizaciones quienes a su vez crean políticas y velan para que cada aeropuerto brinde un servicio de la más alta calidad de la mano de los avances tecnológicos y la innovación.

Sobre este tema, Colombia se ha venido en fortalecimiento en los últimos años. Adicionalmente, con la firma de tratados aerocomerciales y de libre comercio, son muchos retos que enfrenta el país en materia principalmente aeroportuaria. 
Actualmente, el Aeropuerto Internacional Camilo Daza de Cúcuta, Norte de Santander-Colombia, se encuentra en proceso de modernización, cuyas obras elevarán el servicio a un nivel IATA C. Una parte esencial dentro de toda esta fase, es conocer con exactitud y entender los requerimientos de los usuarios que transitan a través del mismo.

Es así como se realizó una extensa revisión bibliográfica, en busca de un método que sirviera de base para los administradores en cuanto al direccionamiento de los recursos y estrategias para elevar los niveles de satisfacción de los usuarios de este aeródromo. Se encontró que el análisis jerárquico de procesos ha sido empleado en diversas áreas de la aviación, debido a su facilidad de adaptación y de desarrollo, arrojando los resultados esperados por los investigadores.

En esta investigación se implementó el análisis jerárquico de procesos difuso a fin de dar manejo a información con difusificidad determinística a objeto de obtener pesos cuantitativos representando la importancia de cada criterio.

\section{ANTECEDENTES}

Muchas investigaciones han sido recientemente llevadas a cabo en materia aeroportuaria. Michelle Carvalho Da Silva junto con Anderson Correia (2008), proponen una metodología para evaluar los niveles de servicio ofrecidos en el aeropuerto de Guarulhos, Sao Paulo. Su estudio se centró en la medición de la importancia asignada a varios componentes del terminal de pasajeros usando el método AHP tradicional. Su investigación destacó como componente más primordial el área de Check-in.

Por otra parte, Yu-Hern Chang, Chien-Hang Cheng y Tong-Chi Wang (2003), llevaron a cabo una Evaluación del Rendimiento de los Aeropuertos Internacionales del este de Asia, donde emplearon Análisis Jerárquico Difuso para asignar los pesos a los criterios y TOPSIS para ordenar el desempeño de cada uno. Como resultado de este trabajo, el Aeropuerto Internacional de Changi, fue catalogado como el mejor dentro de un grupo de 10 , con base en 16 sub-criterios analizados.

El presente estudio, empleó el Análisis Jerárquico de Procesos Difuso, a fin de tolerar la incertidumbre y vaguedad de los juicios humanos, como herramienta para determinar y ordenar los componentes aeroportuarios del caso.

\section{METODOLOGÍA}

El proceso Analítico Jerárquico (Analytic Hierarchy Process, AHP) es una técnica de decisión multi-criterio propuesta por Thomas L. Saaty (70's) que permite que el decisor pueda estructurar un problema multi-criterio en forma visual, mediante la construcción de una jerarquía de atributos. Una vez definida, se realizan comparaciones a pares entre dichos elementos, atribuyéndole valores numéricos a las preferencias señaladas por las personas seleccionadas para estudiar la decisión.

En la mayoría de situaciones donde está involucrada la toma de decisiones, influyen intereses contrapuestos, elementos de incertidumbre, componentes difícilmente valorables, información imprecisa, entre otros, que conllevan a cometer fallos en el tratamiento de los datos del problema en cuestión.

Zadeh (1960's) introdujo por primera vez el término de conjunto borroso, con el fin de manejar la imprecisión de los juicios humanos. Esta teoría ha sido incorporada desde su aparición y aceptación, en muchas metodologías relacionadas no sólo con la toma de decisiones sino en áreas como taxonomía, lógica, teoría de control, psicología, medicina, etc. En la literatura científica, es posible encontrar varias aplicaciones de la lógica difusa en problemas de análisis multi-criterio, que involucran la consideración de varios juicios de expertos. Bezdek (1978), Tanino (1984), Buckley (1985), Van Laarhoven y Pedrycz (1983), Chang (1996), entre otros, son algunos de los autores que han combinado la teoría de conjuntos borrosos en trabajos de análisis de decisión, los cuales han mostrado tener más precisión que las teorías clásicas en general.

Este trabajo investigativo contempló las siguientes fases:

1. Recopilación de Información.

2. Estructuración del problema.

3. Realización de las comparaciones.

4. Fusificación de los datos.

5. Procesamiento y análisis de resultados.

\subsection{Estructuración del problema}

La primera fase contemplada dentro del AHP es la jerarquización del problema de decisión, la cual es representada mediante un árbol de dependencia. Allí se detalla la meta general, junto con las alternativas a ser evaluadas y entre estos dos, los 
criterios/sub-criterios los cuales son los parámetros a tener en cuenta para realizar dicha evaluación. En la figura 1, se detalla el árbol jerárquico empleado en esta investigación:

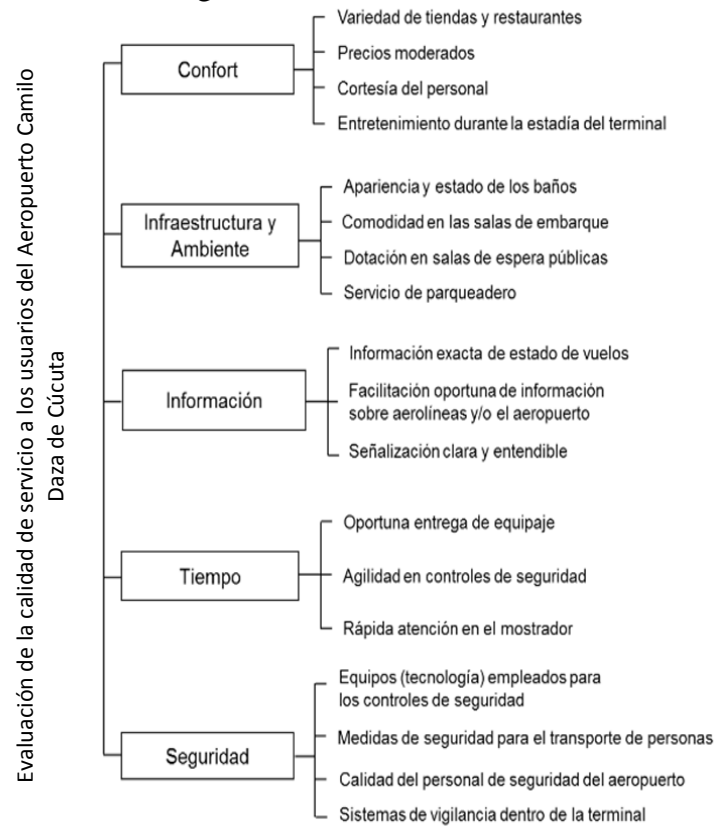

Fig. 1. Árbol de jerarquía, propuesto por el autor.

\subsection{Realización de las comparaciones}

Una encuesta fue aplicada en las instalaciones del Aeropuerto Camilo Daza, a 120 pasajeros de embarque de vuelos nacionales. Esta encuesta fue realizada durante los meses de mayo y junio (2012), a diferentes horas picos del día, con el propósito de captar de la mejor manera las percepciones de los usuarios.

En la encuesta se preguntó a los pasajeros, sobre qué tan importante era un criterio respecto a cada uno de los otros y asimismo con los sub-criterios. Esto con el fin de construir una matriz de comparación en la cual cada celda contiene la magnitud de importancia de la comparación. En la Tabla 1 se aprecia un ejemplo de lo anterior:

\section{Tabla 1: Matriz de comparación de los criterios} principales. Elaboración propia.

\begin{tabular}{|c|c|c|c|c|c|}
\hline & Información & Infraestruc & Confort & Seguridad & Tiempo \\
\hline Información & 1 & $1 / 7$ & 7 & $1 / 5$ & $1 / 7$ \\
\hline Infraestructura & 7 & 1 & 7 & $1 / 9$ & 7 \\
\hline Confort & $1 / 7$ & $1 / 7$ & 1 & 7 & $1 / 7$ \\
\hline Seguridad & 5 & 9 & $1 / 7$ & 1 & 9 \\
\hline Tiempo & 7 & $1 / 7$ & 7 & $1 / 9$ & 1 \\
\hline
\end{tabular}

\subsection{Conversión a números difusos.}

Para realizar la conversión de los números exactos a difusos, se empleó la escala expuesta en la Tabla 2 .
Tabla 2: Tabla de conversión de variables exactas a números triangulares difusos.

\begin{tabular}{|l|c|c|c|}
\hline $\begin{array}{c}\text { Escala lingüística de } \\
\text { importancia }\end{array}$ & $\begin{array}{c}\text { Número } \\
\text { Exacto }\end{array}$ & $\begin{array}{c}\text { Escala } \\
\text { triangular Difusa }\end{array}$ & $\begin{array}{c}\text { Escala } \\
\text { triangular } \\
\text { recíproca difusa }\end{array}$ \\
\hline Igual Importancia & 1 & $(1,1,3)$ & $(1 / 3,1,1)$ \\
\hline Moderada Importancia & 3 & $(1,3,5)$ & $(1 / 5,1 / 3,1)$ \\
\hline Fuerte Importancia & 5 & $(3,5,7)$ & $(1 / 7,1 / 5,1 / 3)$ \\
\hline Muy fuerte Importancia & 7 & $(5,7,9)$ & $(1 / 5,1 / 7,1 / 9)$ \\
\hline Extrema Importancia & 7 & $(7,9,9)$ & $(1 / 7,1 / 9,1 / 9)$ \\
\hline
\end{tabular}

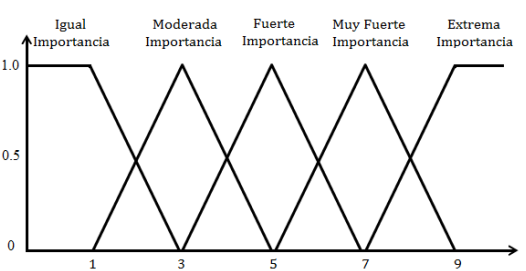

Fig. 2. Escala lingüística de importancia relativa.

La tabla 3 representa la manera como la comparación de los criterios principales eran expresada en números triangulares difusos:

Tabla 3: Matriz difusa de la comparación de
criterios principales. Elaboración propia.
\begin{tabular}{|c|c|c|c|c|c|c|c|c|c|c|c|c|c|c|c|c|}
\hline Informacion & Infraestructura & \multicolumn{3}{|c|}{ Confort } & \multicolumn{5}{|c|}{ Seguridad } & \multicolumn{3}{c|}{ Tiempo } \\
\hline Informacion & 1 & 1 & 3 & $1 / 9$ & $1 / 7$ & $1 / 5$ & 5 & 7 & 9 & $1 / 7$ & $1 / 5$ & $1 / 3$ & $1 / 9$ & $1 / 7$ & $1 / 5$ \\
\hline Infraestructura & 5 & 7 & 9 & 1 & 1 & 3 & 5 & 7 & 9 & $1 / 9$ & $1 / 9$ & $1 / 7$ & 5 & 7 & 9 \\
\hline Confort & $1 / 9$ & $1 / 7$ & $1 / 5$ & $1 / 9$ & $1 / 7$ & $1 / 5$ & 1 & 1 & 3 & 5 & 7 & 9 & $1 / 9$ & $1 / 7$ & $1 / 5$ \\
\hline Seguridad & 3 & 5 & 7 & 7 & 9 & 9 & $1 / 9$ & $1 / 7$ & $1 / 5$ & 1 & 1 & 3 & 7 & 9 & 9 \\
\hline Tiempo & 5 & 7 & 9 & $1 / 9$ & $1 / 7$ & $1 / 5$ & 5 & 7 & 9 & $1 / 9$ & $1 / 9$ & $1 / 7$ & 1 & 1 & 3 \\
\hline
\end{tabular}

Este procedimiento se realiza con todos y cada uno de los grupos de los sub-criterios.

\subsection{Procesamiento y análisis de los resultados}

Una vez construidas las 6 matrices difusas para cada encuestador se procede a realizar el procedimiento matemático de la siguiente manera (Buckley, 1985):

- Promedio de matrices: Las matrices difusas se comparan por medio de la ecuación 1 :

$\tilde{a}_{i j}=\left(\tilde{a}_{i j}^{1} \otimes \tilde{a}_{i j}^{2} \otimes \cdots \otimes \tilde{a}_{i j}^{1}\right)^{1 / n}$

Donde, $\tilde{a}_{i j}, \tilde{a}_{i j}^{1}, \tilde{a}_{i j}^{2}, \ldots, \tilde{a}_{i j}^{n}$ son los juicios expresados por cada evaluador, representado en números triangulares difusos y $n$ es el total de las muestras tomadas

- Cálculo de la media geométrica de las comparaciones usando la ecuación 2:

$$
\tilde{r}_{i}=\left(\tilde{a}_{i 1} \otimes \tilde{a}_{i 2} \otimes \cdots \otimes \tilde{a}_{i n}\right)^{1 / n}
$$


- Obtención de la matriz de pesos difusa a partir de la ecuación 3:

$$
\tilde{w}_{i}=\tilde{r}_{i} \otimes\left(\tilde{r}_{1} \otimes \cdots \otimes \tilde{r}_{n}\right)^{1 / n}
$$

- Finalmente, los pesos difusos de prioridad son convertidos nuevamente en números exactos por el método del centro de área, según la ecuación 4 :

$$
B N P_{i}=\left\{\left[\left(U R_{i}-L R_{i}\right)+\left(M R_{i}-L R_{i}\right)\right] / 3\right\}+L R_{i}(4)
$$

Una vez obtenido el BNP, (Best Non-fuzzy Performance), los datos se someten a un proceso de normalización, que dará como resultado la clasificación de cada una de los componentes de acuerdo a su importancia. Esto servirá como base para la toma de decisiones por parte de la administración.

\section{CONCLUSIONES Y RECOMENDACIONES}

Los resultados obtenidos de la encuesta realizada a los pasajeros del Aeropuerto Camilo Daza mostraron que el componente más importante sobre todos los evaluados es la Seguridad, seguida de Tiempo, Confort, Infraestructura e Información, respectivamente. Siendo así, se decidió consultar una segunda opinión, esta vez de los expertos, con el fin de confrontar ambas y tener una perspectiva más general.

A los expertos se les aplicó el mismo cuestionario y se realizó el mismo análisis matemático, cuyos resultados se expresan en la tabla 4.

El análisis resultó muy interesante, ya que en este último grupo de encuestados el criterio más importante, al igual que el anterior, fue Seguridad, seguido en cambio de Información.

El aspecto que las personas que comúnmente transitan por las instalaciones aeroportuarias de Cúcuta consideraron como menos importante de todo el conjunto, resultó siendo el segundo componente más importante para los expertos, y ambos coincidieron en que la Seguridad será siempre lo más importante por delante de cualquier otro criterio.
Se recomienda, para futuras investigaciones, plantear el árbol de jerarquía, preferiblemente, con los aspectos más importantes de la manera más resumida posible, para evitar de esta manera fallos al momento de las comparaciones.

Cuando no hay antecedentes relevantes, que ayuden a conducir el proyecto de una manera acertada, sería muy útil llevar a cabo una prueba piloto, para detectar errores a tiempo y realizar los correctivos respectivos.

\section{REFERENCIAS}

Saaty L., S. y VargasG., L. (2012). Models, Methods, Concepts \& Applications of the Analytic Hierarchy Proces. Springer, Segunda edición, Nueva York.

Llamazares R., F. (2011). Los Métodos de Desición Multicriterio y su Aplicación al Análisis del Desarrollo Local, Esic Editorial, Primera edición, España.

Fernández S., E. (2010). Administración de empresas. Un enfoque Interdisciplinar, Paraninfo, Primera edición, España.

Herrera C., J. (2008). Toma de decisiones en simulación basadas en la teoría de decisión multicriterio, Tesis de Maestría. Postgrado en Investigación en Informática. Universidad Complutense, Madrid.

Smith Q., R., Jaramillo A., G., Vélez U., J., Botero V., y Caballero H. (2005). Desarrollo de técnicas para el manejo de incertidumbre e imprecisión en problemas de decisión con múltiples objetivos, Dirección de Investigadores sede Medellín, Facultad de Minas, Universidad Nacional de Colombia.

Gachet O., F. (2002). La Huella Ecológica: Teoría, Método y Tres aplicaciones al Análisis Económico, Ediciones Abya-yala, Primera edición, Quito.

Cabañete C., A. (1998). Toma de decisiones. Análisis y entorno organizativo, Ediciones UPC, Primera edición, España.

Young-Jou L. y Ching-Lai., H. (1996). Fuzzy Multiple Objective Decision Making, Springer.

Mendel, J. (1995). "Fuzzy Logic Systems for Engineering: A tutorial". Proceedings of the IEEE, Vol. 83 (3), pp. 345-377.

Zadeh L., (1975). Fuzzy Sets and their applications to cognitive and decision processes, Academic Press Inc., $1^{\text {ra }}$ Edición. 


\section{SITIOS WEB}

Serhat A., y Cengiz C., (2011). A modified Fuzzy Analytic Hierarchy Process based Multicriteria Decision Making methodology for assessing ecommerce website quality: A case study in Turkey, Proceedings of the World Congress on Engineering. Vol. II. London.

http://iaeng.org/publication/WCE2011/WCE2011_ pp1174-1178.pdf

Mei-Ling, C., Wei-Mao C., y James J., L., (2009). Evaluating corporate image and reputation using fuzzy MCDM approach in airline market. Taiwan.

http://isahp.org/2009Proceedings/Final_Papers/17 _Chuang_FuzzyMCDMEvaluatingCorporateIm ageReputationAirlineMarket_REV_FIN.pdf

Bandeira, M., y Correira, A. (2008). Analysis of the degree of importance of the airport passenger terminal components in São Paulo/Guarulhos International Airport, Brazil.

Osorio G., J., Herrera U., F., y Vinasco A., (2008). Modelo para la evaluación del desempeño de los proveedores utilizando AHP, Colombia.

http://www.icesi.edu.co/biblioteca_digital/bitstrea m/item/810/2/Modelo_gestion_proveedores_uti lizando_AHP_difuso.PDF

Geimba de L., M., Côrtes P., C., Piratelli C., Neyra B., Mischel., y Correira, A. (2007). Using
Analytic Hierarchy Process for analysis and choice of Brazilian cargo Airlines, Brazil.

http://www.isahp.org/2007Proceedings/Papers/Wor king\%20Sessions/Suppliers\%20Selection/Brazi lian\%20Cargo\%20Airlines\%20Selection.pdf

Ting-Ya, H., Shih-Tong L., y Gwo-Hshiung, T., (2004). Fuzzy MCDM approach for planning and design tenders selection in public office buildings, National Cental University, Taiwan.

http://www.knu.edu.tw/lecture/\%E6\%AD\%B7\%E5 $\% \mathrm{~B} 9 \% \mathrm{~B} 4 \% \mathrm{E} 6 \% 95 \% 99 \% \mathrm{E} \% \mathrm{AD} \% \mathrm{~B} 8 \% \mathrm{E} 8 \% \mathrm{~B}$ 3\%87\%E6\%96\%99/2009-05-

13\%Е5\%B9\%B4\%Е7\%A0\%94\%Е7\%A9\%B6 $\%$ Е6\%96\%B9\%Е6\%B3\%95\%Е5\%8F\%83\%Е8 $\% 80 \% 83 \%$ E6\%96\%87\%E7\%8D\%BB/Exa.5.13 .09/Fuzzy\%20MCDM\%20approach\%20for\%2 Oplanning\%20and\%20design $\% 20$ tenders $\% 20$ se lection\%20in\%20public\%20office\%20building s.pdf

Yu-Hern, C., Chieng-Hang C., y Tong-Chi W., (2003). Performance evaluation of International Airports in the region of east Asia, Taiwan.

http://www.easts.info/2003proceedings/papers/021 3.pdf

Tabla 4. Cuadro resumen y clasificación final de los criterios y sub-criterios. (Fuente: elaboración propia).

\begin{tabular}{|c|c|c|c|c|c|c|c|c|c|c|}
\hline Criterios y Sub-criterios & & Pesos P & ales & & & Totales & & BNP & BNP NORMALIZADO & CLASIFICACIÓN \\
\hline Información & 0,0721 & 0,1601 & 0,3749 & & & & & 0,2024 & 0,1700 & 2 \\
\hline Señalización clara y entendible & & 0,0544 & 0,1226 & 0,3314 & 0,0039 & 0,0196 & 0,1242 & 0,0493 & 0,0242 & 13 \\
\hline Información exacta de estado de vuelos & & 0,2195 & 0,5724 & 1,3376 & 0,0158 & 0,0916 & 0,5015 & 0,2030 & 0,0997 & 3 \\
\hline Facilitación oportuna de información & & 0,1284 & 0,3051 & 0,8166 & 0,0093 & 0,0488 & 0,3061 & 0,1214 & 0,0596 & 6 \\
\hline Confort & 0,0372 & 0,075 & 0,1786 & & & & & 0,0969 & 0,0814 & 5 \\
\hline Variedad de tiendas y restaurantes & & 0,1523 & 0,3525 & 0,813 & 0,0057 & 0,0264 & 0,1452 & 0,0591 & 0,0290 & 10 \\
\hline Precios moderados & & 0,1241 & 0,2873 & 0,6604 & 0,0046 & 0,0215 & 0,1179 & 0,0480 & 0,0236 & 14 \\
\hline Cortesía del personal & & 0,0953 & 0,2231 & 0,4966 & 0,0035 & 0,0167 & 0,0887 & 0,0363 & 0,0178 & 15 \\
\hline Entretenimiento durante la estadía & & 0,0611 & 0,1371 & 0,3405 & 0,0023 & 0,0103 & 0,0608 & 0,0245 & 0,0120 & 17 \\
\hline Infraestructura y Ambiente & 0,0478 & 0,102 & 0,2348 & & & & & 0,1282 & 0,1077 & 3 \\
\hline Apariencia y estado de los baños & & 0,2119 & 0,4719 & 0,9722 & 0,0101 & 0,0481 & 0,2283 & 0,0955 & 0,0469 & 7 \\
\hline Comodidad en las salas de embarque & & 0,1403 & 0,3049 & 0,662 & 0,0067 & 0,0311 & 0,1554 & 0,0644 & 0,0316 & 9 \\
\hline Servicio de parqueadero & & 0,0321 & 0,0638 & 0,1528 & 0,0015 & 0,0065 & 0,0359 & 0,0146 & 0,0072 & 18 \\
\hline Dotación en salas de espera públicas & & 0,0784 & 0,1594 & 0,374 & 0,0037 & 0,0163 & 0,0878 & 0,0359 & 0,0176 & 16 \\
\hline Tiempo & 0,0402 & 0,0805 & 0,1927 & & & & & 0,1045 & 0,0878 & 4 \\
\hline Oportuna entrega de equipaje & & 0,1118 & 0,2566 & 0,674 & 0,0045 & 0,0207 & 0,1299 & 0,0517 & 0,0254 & 12 \\
\hline Rápida atención en el mostrador & & 0,1675 & 0,4424 & 1,0688 & 0,0067 & 0,0356 & 0,2060 & 0,0828 & 0,0406 & 8 \\
\hline Agilidad en los controles de seguridad & & 0,1234 & 0,3011 & 0,7407 & 0,0050 & 0,0242 & 0,1427 & 0,0573 & 0,0281 & 11 \\
\hline Seguridad & 0,2805 & 0,5824 & 1,1123 & & & & & 0,6584 & 0,5531 & 1 \\
\hline Sistemas de vigilancia en la terminal & & 0,0594 & 0,1255 & 0,3165 & 0,0167 & 0,0731 & 0,3520 & 0,1473 & 0,0723 & 5 \\
\hline Equipos tecnológicos empleados & & 0,0774 & 0,1742 & 0,4123 & 0,0217 & 0,1015 & 0,4586 & 0,1939 & 0,0952 & 4 \\
\hline Calidad del personal de seguridad & & 0,2166 & 0,5116 & 1,1536 & 0,0608 & 0,2980 & 1,2831 & 0,5473 & 0,2688 & 1 \\
\hline $\begin{array}{l}\text { Medidas de seguridad para el transporte } \\
\text { de personas }\end{array}$ & & 0,0788 & 0,1887 & 0,4317 & 0,0221 & 0,1099 & 0,4802 & 0,2041 & 0,1002 & 2 \\
\hline
\end{tabular}

